\title{
RESUMEN
}

El presente proyecto pretende analizar el impacto que tienen los conocimientos académicos (CA) y la inteligencia emocional (IE) como elementos de la formación emprendedora en la importancia percibida de planeación estratégica $(\mathrm{PE})$ como factor que incide en el desarrollo y consolidación de las Micro, Pequeñas y Medianas Empresas (MiPyMEs). Asimismo, se resalta la importancia que se fomente el emprendimiento desde temprana edad ya que la población joven que tenga conocimientos teóricos adquiridos en su nivel profesional aunado a la experiencia de emprender da pauta a un nivel emprendedor mayor en México. Como metodología se realizó un análisis de correlación para conocer la relación causal entre las variables de estudio, así como un modelo de regresiones lineales, a fin de identificar la capacidad explicativa de las variables CA e IE sobre la variable importancia percibida de la $\mathrm{PE}$, en los emprendedores del sector restaurantero de la Zona Metropolitana de Guadalajara (ZMG)

Palabras clave: Consolidación MiPyMEs, emprendimiento, emprendedores, educación superior

\section{ELEMENTOS DE FORMACIÓN EMPRENDEDORA EN EL USO DE HERRAMIENTAS PARA LA CONSOLIDACIÓN DE MIPYMES: CASO DEL SECTOR RESTAURANTERO}

\author{
José Rosario Lara Salazar ${ }_{1}$ / Itzel Alejandra Lara Manjarrez / Alejandro Campos Sánchez $_{3}$
}

\author{
Fecha de recepción: 20 de enero 2021 \\ Fecha de aceptación: 06 de abril 2021
}

DOI: https://doi.org/10.22370/riace.2021.10.1.2831

1 Centro Universitario de Ciencias Económico-Administrativas, Universidad de Guadalajara, Zapopan, México. jose.larasalazar09@gmail.com

2 Centro Universitario de Ciencias Económico-Administrativas, Universidad de Guadalajara, Zapopan, México. itzelara1@hotmail.com

3 Centro Universitario de Ciencias Económico-Administrativas, Universidad de Guadalajara, Zapopan, México.a.campos@cucea.udg.mx 


\title{
ELEMENTS OF ENTREPRENEURIAL TRAINING IN THE USE OF TOOLS FOR THE CONSOLIDATION OF MSMES: CASE OF THE RESTAURANT SECTOR
}

\begin{abstract}
This project aims to analyze the impact of academic knowledge (AK) and emotional intelligence (EI) as elements of entrepreneurial training on the perceived importance of strategic planning (SP) as a factor that affects the development and consolidation of Micro, Small and Medium Enterprises (MSMEs). Likewise, the importance of promoting entrepreneurship from an early age is highlighted since the young people who have acquired theoretical knowledge in their professional level coupled with the experience of entrepreneurship gives a guideline to a higher entrepreneurial level in Mexico. As a methodology, a correlation analysis was carried out to know the causal relationship between the variables, as well as a linear regression model, to identify the explanatory capacity of the AK and El as a variable perceived importance of SP, in the entrepreneurs of the restaurant sector of the Guadalajara Metropolitan Area (GMA).
\end{abstract}

Keywords: MSMEs consolidation, entrepreneurship, entrepreneurs, higher education 


\section{INTRODUCCIÓN}

En la actualidad, las MiPyMEs constituyen un eje vertical en la economía nacional, debido a que son el principal ingreso de millones de familias en México las cuales representan el $99.8 \%$ del total de empresas en el país, con más de $52 \%$ de aporte al PIB nacional (Ordaz-Díaz, 2019). Por tal motivo, se hace hincapié en la importancia de estas empresas, ya que su permanencia se deriva en un crecimiento económico para la nación.

En este sentido, es importante conocer de manera profunda los principales factores que impactan de manera positiva el proceso de desarrollo y consolidación de estas empresas en México, ya que esto se traduce en una mayor permanencia y longevidad de las organizaciones. De manera que, según Corredoira y Kimberly (2006) la consolidación es el impulso que tiene una empresa para la búsqueda de oportunidades con el objetivo de aprovechar nuevas tecnologías, explorar nuevas iniciativas regulatorias e introducir economías de escala y alcance en una industria.

Siguiendo con lo anterior, uno de los factores identificados como sustanciales para la consolidación empresarial es el hecho de contar con una adecuada y eficiente $\mathrm{PE}$. En este sentido, se considera que los CA constituyen un elemento de conciencia respecto a la importancia de planear de manera adecuada y formal la estrategia a seguir en el proyecto de emprendimiento o en el negocio marcha.

Por otra parte, se considera también que un elemento cognitivo como la IE, influye de manera positiva en la percepción de la importancia, por parte del emprendedor, de contar con un proceso adecuado de PE para ejecutar con éxito el proceso de emprendimiento y la futura gestión del proyecto.

Es así como se plantea que en un proceso emprendedor, la sumatoria de las dimensiones de CA y la IE afectan de manera positiva la percepción de un emprendedor sobre la importancia de la PE en el proceso de crear una empresa, y por ende en su posible permanencia y consolidación futura.

Fretschener y Weber (2013) mencionan que la educación empresarial impacta directamente en la intención emprendedora de los estudiantes de licenciatura dando como resultado un mayor porcentaje de consolidación empresarial de dichos universitarios que se han convertido en pequeños empresarios.

Del mismo modo, este estudio pretende mostrar un panorama de la situación actual generada a causa de la emergencia sanitaria a causa del virus COVID-19 a nivel mundial y cómo esta ha impactado al sector empresarial poniendo a prueba los CA de los emprendedores, así como su nivel de IE para saber qué decisiones son las más benéficas para su organización y qué adecuaciones realizar a su PE original.

Así pues, el objetivo del presente estudio es analizar el impacto de los CA adquiridos durante la etapa formativa del emprendedor, así como los elementos de su IE en la importancia percibida de la PE de sus proyectos, en uno de los sectores más golpeados por la pandemia pero a la vez, uno de los más importantes en la vida económica de la ZMG. 
A través de una metodología cuantitativa, se llevará a cabo un análisis de correlación para conocer la relación causal entre las variables de estudio. También se propone un modelo de regresiones lineales, a fin de identificar la capacidad explicativa de las variables CA e IE sobre la variable importancia percibida de la PE, en los emprendedores del sector restaurantero de la ZMG.

\section{Sector restaurantero en la ZMG}

Según el Directorio Estadístico Nacional de Unidades Económicas (DENUE) en la ZMG se encuentra un total de 24,202 restaurantes (Instituto Nacional de Estadística y Geografía, 2021). La industria restaurantera de la ZMG se ha caracterizado por ser un sector importante, ya que trae consigo beneficios económicos tales como la generación de empleos, así como un aporte al turismo de la región.

Del mismo modo, en el sitio web de la Cámara Nacional de la Industria Restaurantera y de Alimentos Condimentados de Jalisco (CANIRAC) (2020) menciona que la ZMG junto con Puerto Vallarta concentra la mayor cantidad de restaurantes a nivel nacional por lo que se puede distinguir la trascendencia de dicha industria para el país.

En este tenor, se puede hacer notar la importancia del sector restaurantero para la economía del estado, por lo que la permanencia y consolidación de esta resulta imperativa para la sociedad. Desafortunadamente, la industria restaurantera es uno de los sectores más afectados por la pandemia por COVID-19 suscitada a nivel mundial, ya que sus ventas han caído hasta en un $90 \%$ lo cual ha dañado considerablemente a los emprendedores de la zona (Mata-Loera, 2020). Por tal motivo, los pequeños negocios, se vieron forzados a adquirir plataformas como Rappi y Uber Eats para poder distribuir sus productos o alimentos a sus consumidores, ya que la mayoría de los negocios se encontraban imposibilitados para trabajar de forma ordinaria por lo que la forma más sencilla de poder seguir laborando era por medio de la colaboración con dichas plataformas.

\section{Los emprendimientos ante la emergencia sanitaria por COVID-19}

La pandemia ocasionada por coronavirus a nivel mundial ha desencadenado una serie de sucesos que no pudieron prevenirse de forma anticipada como es una baja fuerte en la Economía nacional. Sobre esto, la Organización para el Desarrollo y la Cooperación Económica (OCDE) informó que el PIB de México tendrá una caída de $10.2 \%$ a causa en mayor medida por las afectaciones surgidas en el sector turístico, las exportaciones, así como la caída del precio del petróleo lo cual genera pérdidas importantes para la economía mexicana (Forbes, 2020).

Adicionalmente, según datos del Instituto Nacional de Estadística y Geografía (INEGI) muestra que el $93.2 \%$ de las empresas en México presentaron al menos 
un tipo de afección a causa de la contingencia por coronavirus. Entre dichas afectaciones la mayor fue la disminución de ingresos con un $91.3 \%$ a la baja, así como un descenso en la demanda de $72.6 \%$ a nivel nacional.

Del mismo modo, se hizo un análisis por medio de la "Encuesta sobre el Impacto Económico Generado por COVID-19 en las Empresas (ECOVID-IE)" el cual arrojó que se prevé que más de la mitad de las organizaciones encuestadas tuvieron que realizar paros técnicos o cierres temporales como medidas preventivas para evitar la propagación del virus dentro del trabajo (Instituto Nacional de Estadística y Geografía, 2020).

Teniendo lo anterior en consideración, se puede hacer notar las malas condiciones económicas en las que se encuentra el país debido a la incertidumbre y entornos cambiantes que se han suscitado lo cual se traduce en un freno e impacto negativo directo para las MiPyMEs y los emprendedores.

Así pues, la desaceleración económica ha forzado a los empresarios a reestructurar el modelo de negocio existente y adaptarse a las nuevas realidades para garantizar la permanencia y continuidad de las empresas. Hecha tal salvedad, la tecnología se ha convertido en elemento clave para brindar soluciones a los clientes sin descuidar el bienestar de estos. En este tenor, la consolidación de las organizaciones se ha visto más comprometida debido a los factores externos que se han suscitado de manera inesperada y poco previsible.

Por tal motivo, la aparición de plataformas digitales ha generado nuevas brechas en las que es menester que las empresas realicen una reconfiguración de procesos, así como la introducción de mejores prácticas que brinden un cambio positivo para la misma, que otorguen mayor visibilidad y por ende más posibilidades de ser adquiridos por los consumidores (Microsoft Latinoamérica, 2020).

\section{La planeación estratégica en la consolidación de los emprendimientos}

La consolidación de un emprendimiento para fines de este estudio se conceptualiza como fue mencionado con anterioridad como la necesidad que una organización tiene para encontrar nuevas oportunidades y con ello cumplir sus objetivos a través de herramientas diversas como las tecnológicas y con ello posicionarse en el sector industrial de su correspondencia.

Es necesario destacar, que definir el término consolidación no es sencillo pues el punto para alcanzar dicha etapa puede ser variable de acuerdo con la zona geográfica. Sin embargo, Bolin (2015) y Senior (2016) mencionan que, de acuerdo con un análisis a profundidad, las ganancias económicas son el punto más relevante que lleva a una empresa consolidarse. Por otro lado, la consolidación puede definirse como el proceso en que una empresa pueda subsistir al menos cinco años (Schofield, 2015) y que tenga buena solvencia financiera lo que conllevará según Jonckheere (2015) a que la empresa no dependa necesariamente de apalancamiento financiero de terceros. 
Destacando la importancia de lo dicho, Kaiser (2011) establece un parámetro en el que una empresa puede considerarse consolidada a partir de los 4 años de su creación y desarrollo de operaciones. Partiendo de dicha premisa, el modelo de Churchill y Lewis (1983), analizan los 5 pasos (véase tabla 1) desde la creación de una empresa hasta el punto de su consolidación.

Tabla 1.- Cinco etapas del desarrollo de una empresa

\begin{tabular}{|l|l|}
\hline \multicolumn{1}{|c|}{ Nombre } & \multicolumn{1}{c|}{ Descripción } \\
\hline Existencia & Obtención de clientes \\
\hline Supervivencia & Existe viabilidad, sin embargo, se carece de certidumbre para su futuro \\
\hline Éxito & Se han obtenido logros. Es necesario explotarlos \\
\hline Despegue & Crecimiento de la empresa \\
\hline Consolidación & Tienen base fuerte; puede competir en el mercado \\
\hline
\end{tabular}

Fuente: elaboración propia con base en Churchill y Lewis (1983)

Como se puede analizar en la tabla anterior, las etapas de desarrollo de una empresa están claramente diferenciadas cada una con respecto a las otras. Primeramente, en la existencia se busca la obtención de clientes, de lo cual Guadarrama y Rosales (2015) mencionan que suele ser el paso más difícil de lograr debido a que, el producto y/o servicio ofertado es altamente probable que exista en el mercado y cabría preguntarse la razón por la cual un cliente debería preferir la oferta dada por la empresa de reciente creación a una que ya sea conocida. En esta etapa suele haber mucha preocupación por parte del empresario, ya que su nivel de pericia puede llegar a ser bajo respecto con la competencia.

Una vez creada la empresa, sigue el proceso el cual suele ser el más delicado de las 5 etapas, el de la supervivencia, pues aquí, puede ser variable la esperanza de vida que tiene una empresa en México de acuerdo con el sector. Como fue mencionado anteriormente, según el INEGI (2020) teniendo en promedio los sectores manufactureros, comerciales y servicios privados no financieros, la esperanza de vida de las empresas es de 7.8 años (véase figura 1), aunque esto no es sinónimo que es necesario pasar por dicho periodo para poder consolidarse. El proceso de consolidación puede ser antes o después de los datos establecidos por el INEGI. 
Figura 1.- Esperanza de vida de las PyMEs en México

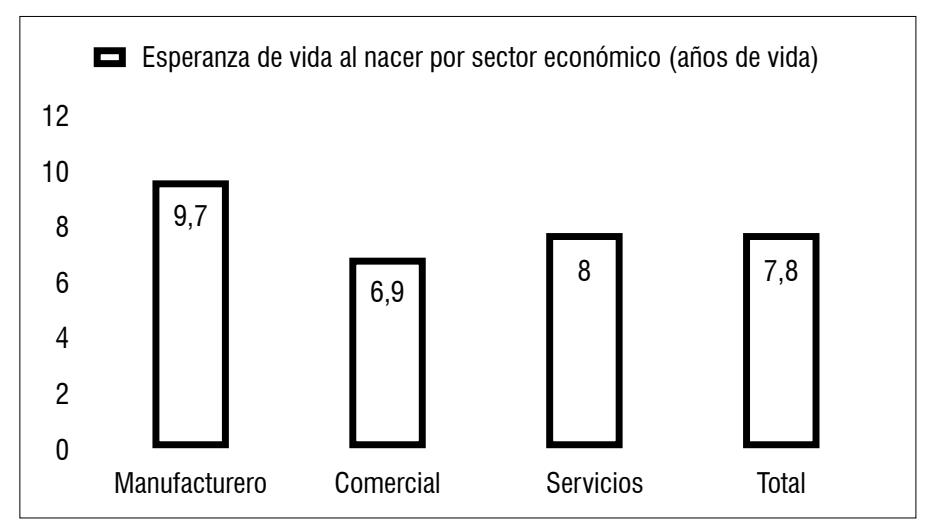

Fuente: Instituto Nacional de Estadística y Geografía (2020)

Continuando con la tercera etapa, se muestra el éxito. Las empresas han logrado establecer un número de ventas suficientes en el cual, no es necesario aportar de capital propio sino del mismo capital que está ingresando a la empresa. En combinación con esto, se puede ejemplificar la ampliación del número de clientes o la expansión mediática de la empresa a través de medios difusivos como las redes sociales.

En la parte final, se encuentra la consolidación, en la cual la empresa ha podido establecerse con una cartera de clientes establecida, con finanzas normalmente estables, y con un nivel de conocimiento y pericia más elevado por parte del empresario.

Lussier (1992) define que la consolidación de la empresa se mide por el tiempo que esta lleve en el mercado y que dé pauta a pensarse que seguirá así. Tal acción ejerce una alta posibilidad que suceda si dentro de la empresa se lleva un buen control financiero, similar a lo dicho por Coleman (1994) sobre el manejo financiero que una empresa debe tener como punto importante.

De tal forma, se concluye que la consolidación de una empresa se ve afectada por diversos factores, pero sin duda alguna, uno elemental según Wen-hsin et al (2015) es el dinero que fluya dentro de la organización, mismo que puede verse perdido muchas veces por falta de pericia de planeación por parte de los emprendedores.

Dicho lo anterior, para una consolidación efectiva, se necesita de una organización y visión por lo que, en este punto, la PE funge un papel primordial. De acuerdo con Ronmark (2005) la PE brinda información certera al emprendedor para que el nivel de consolidación de su empresa sea aún más cercano a concretarse, así como aquellos que no utilizan la PE son altamente sensibles a caer en el fracaso empresarial.

Además, la PE es establecida como la aplicación de esfuerzos para que los recursos sean optimizados, así como para asegurar que las operaciones de las organizaciones generen un costo inferior con un servicio de alta calidad en añadidura 
(Kayuni, 2016) lo que muestra con claridad la importancia de la implementación de la PE pues el tiempo y los recursos tienen un nivel más probable de optimizarse si se ejerce una planeación con anticipación.

Por ello, la PE de acuerdo con Walter y Pando (2014) es un medio para fortalecer el alineamiento de la acción con los objetivos de la empresa; es decir, son conceptos, procedimientos y herramientas para el análisis sistemático y la revisión de la orientación de la acción.

Así pues, para generar una buena PE es relevante considerar no solo aspectos externos como los factores políticos o económicos sino también aquellos factores internos que el mismo emprendedor tiene como la percepción de sus capacidades y oportunidades. El Global Entrepreneurship Monitor (GEM) aporta información a nivel mundial y nacional sobre datos del emprendimiento que cada país lleva como se muestra a continuación

Tabla 2.- Auto percepción del emprendedor mexicano

\begin{tabular}{|l|c|c|c|}
\hline & $\begin{array}{c}\text { Porcentaje en } \\
\text { México en 2017 }\end{array}$ & $\begin{array}{c}\text { Porcentaje en } \\
\text { México en 2019 }\end{array}$ & $\begin{array}{c}\text { Media del } \\
\text { porcentaje en el } \\
\text { mundo }\end{array}$ \\
\hline Tasa de oportunidades percibidas & 36.38 & 62.81 & 53.65 \\
\hline Tasa de capacidades percibidas & 50.07 & 70.68 & 58.67 \\
\hline Tasa de temor al fracaso & 28.40 & 47.74 & 41.74 \\
\hline Tasa de intención emprendedora & 13.20 & 16.30 & 23.72 \\
\hline
\end{tabular}

Fuente: elaboración propia con base en Global Entrepreneurship Monitor (2019)

Como se observa en la tabla 2, el uso de una PE es de necesidad apremiante a razón de que en México se supera la tasa media mundial en oportunidades y capacidades percibidas, pero en el momento de querer emplearlas, la tasa al temor al fracaso impacta fuertemente y conlleva que emprendedores no tengan el ímpetu final de emprender o si lo hacen, van más con acciones alineadas a la empírea por encima de una estrategia estructurada dando como consecuencia tener una tasa de intención emprendedora siete puntos porcentuales por debajo de la media global.

Así pues, Bolisani y Bratianu (2018) establecen sobre el momento de la formulación de la PE y el impacto positivo que se tiene en visualizar las ventajas y desventajas sobre el entorno en donde esté operando el emprendimiento como ha sido observado en los resultados del GEM. Finalmente, se destaca que, una PE bien estructurada no genera una infalibilidad en la consolidación de un emprendimiento, pero sí da alta probabilidad que esto suceda. 


\section{Conocimientos académicos}

Los conocimientos y capacidades del emprendedor, para efectos de este estudio, se consideran representados por aquellos CA que el sujeto emprendedor ha recibido durante su etapa formativa y/o de educación formal. Dentro de esos CA se destacan la capacidad de recepción de información sobre las características de un emprendedor, así como sus actitudes tales como la asunción al riesgo 0 la adaptación del cambio ejemplificado con casos de éxito. Aunado a ello, dentro de los CA se menciona la importancia de lo que es un emprendedor y el proceso que este debe llevar a cabo para cumplir el fin del emprendimiento, desarrollo y consolidación de este. Mientras que las capacidades, refieren al aspecto actitudinal en situaciones disyuntivas, explicado directamente como la IE.

Primeramente, Ios CA por parte del emprendedor fungen como parte medular para que estos puedan tener un mayor nivel de éxito empresarial (The European Commission, 2006) esto sucede por la razón que de acuerdo con Green et al. (2014) las instituciones académicas han notado y comprobado que entre mayor sea el conocimiento empresarial que adquieran los futuros emprendedores, mayor es la posibilidad que estos puedan crear empresas a largo plazo y que por ende, el sector privado se acerque con las universidades para establecer convenidos con visión a largo plazo.

Carayannis (2011) menciona que, desde la primera clase de emprendimiento en los Estados Unidos en 1947, han existido más de 2000 cursos en más de 1600 escuelas, lo que elevó de manera sustancial los niveles de emprendimiento en dicho país, lo cual generó al mismo tiempo que se replicara esta actividad en otros países, principalmente latinoamericanos. Es así como con esto, los conocimientos que un emprendedor tiene fomentan a mayor crecimiento del emprendimiento que han realizado pues llevan ideas innovadoras de aplicación de tecnologías o de un mejor entendimiento del entorno en el que se encuentran (Gambardella \& Florence, 2015), causando que el emprendimiento realizado tenga una mayor oportunidad no solo de subsistencia en el mercado, sino de un nivel de consolidación sólido.

Continuando con esta idea, los CA fomentan mayor investigación dentro de un emprendimiento pues buscan que lo aplicado en este mismo, se pueda respaldar con evidencia académica y científica (Hayter \& Link, 2018) pues esto puede elevar el porcentaje de posibilidad que un emprendimiento pueda competir con mayor nivel dentro de un mercado con un nivel competitivo más alto.

Es necesario destacar asimismo según Bartkowiak et al (2020) que dentro del conocimiento adquirido por un emprendedor, se resalta del intelecto del mismo pues con este conocimiento no solo incrementan la posibilidad de consolidación del emprendimiento, sino que le generan valor en el mercado y esto a su vez fomenta la sinergia dentro de la organización con todos los colaboradores para que se incentive a estar en constante capacitaciones dentro y/o fuera de la misma. 
Esto nos lleva a plantear la siguiente hipótesis de investigación:

H1. Los conocimientos académicos del emprendedor influyen positivamente en la importancia percibida de la planeación estratégica.

\section{Inteligencia emocional}

Carter (2015) define la IE como una habilidad aprendida para identificar, experimentar, comprender, y expresar las emociones humanas de manera saludable y productiva. Del mismo modo, Furnham (2012) menciona que la IE es el encanto y la influencia que tiene un individuo, así como las habilidades sociales interpersonales dando como resultado el $80 \%$ del éxito general de la vida de las personas con un solo $20 \%$ para la IE.

En ese tenor, Walters (2018) menciona que la aplicación de la IE ha mostrado que puede ayudar al fomento del desarrollo del liderazgo. Asimismo, de acuerdo con Goleman et al (2013) la adaptabilidad, la identificación de emociones en el momento, el autocontrol, lidiar con situaciones que generen niveles de estrés elevados, influir en otros y el trabajo en equipo es parte necesaria en la IE de un emprendedor.

Para comprender más aún sobre la IE, Mayer et al (2016) mencionan que esta variable está compuesta de siete principios que se muestran en la siguiente tabla:

Tabla 3.- Explicación sobre la inteligencia emocional

\begin{tabular}{|c|c|}
\hline Principio & Explicación del principio \\
\hline $\begin{array}{l}\text { Principio 1: IE es una habilidad } \\
\text { mental }\end{array}$ & $\begin{array}{l}\text { Las personas inteligentes emocionalmente perciben las } \\
\text { emociones de manera precisa; utilizan emociones para facilitar } \\
\text { con precisión el pensamiento; entienden las emociones y sus } \\
\text { significados; manejan sus emociones y la de los demás. }\end{array}$ \\
\hline $\begin{array}{l}\text { Principio 2: IE es mejor medida } \\
\text { como habilidad }\end{array}$ & $\begin{array}{l}\text { Las inteligencias se miden mejor como habilidades, planteando } \\
\text { problemas para que las personas las resuelvan y examinando } \\
\text { los patrones resultantes de respuestas correctas. Debido a que } \\
\text { las personas desconocen qué implica realmente una buena } \\
\text { resolución de problemas, estiman sus habilidades en otras } \\
\text { bases. Estos incluyen una mezcla de autoconfianza general, } \\
\text { autoestima, malentendidos de lo que implica un razonamiento } \\
\text { exitoso y una ilusión }\end{array}$ \\
\hline $\begin{array}{l}\text { Principio 3: La solución } \\
\text { inteligente de los problemas no se } \\
\text { corresponde perfectamente con el } \\
\text { comportamiento inteligente }\end{array}$ & $\begin{array}{l}\text { Hay una distinción significativa entre inteligencia y } \\
\text { comportamiento. El comportamiento de una persona es una } \\
\text { expresión de la personalidad de ese individuo en un contexto } \\
\text { social dado. La personalidad de un individuo incluye motivos } \\
\text { y emociones, estilos sociales, autoconciencia y autocontrol, } \\
\text { todo lo cual contribuye a la consistencia en el comportamiento, } \\
\text { además de la inteligencia. Así pues, las personas } \\
\text { emocionalmente estables, extrovertidas y conscientes pueden } \\
\text { ser emocionalmente inteligentes o no. }\end{array}$ \\
\hline
\end{tabular}




\begin{tabular}{|l|l|}
\hline \multicolumn{1}{|c|}{ Principio } & \multicolumn{1}{|c|}{ Explicación del principio } \\
\hline $\begin{array}{l}\text { Principio 4: El contenido de una } \\
\text { prueba (el área de resolución } \\
\text { de problemas involucrada) debe } \\
\text { especificarse claramente como una } \\
\text { condición previa para la medición de } \\
\text { las capacidades mentales humanas }\end{array}$ & $\begin{array}{l}\text { Para medir bien la inteligencia emocional, las pruebas deben } \\
\text { tomar muestras del tema necesario; el contenido de la prueba } \\
\text { debe cubrir el área de resolución de problemas. Una vez que se } \\
\text { establece el contenido de la prueba, la prueba se puede utilizar } \\
\text { para identificar las habilidades mentales de una persona. Las } \\
\text { capacidades de resolución de problemas de las personas se } \\
\text { reflejan en la estructura correlacional (o covarianza) de las } \\
\text { respuestas que hacen a los ítems de la prueba. }\end{array}$ \\
\hline $\begin{array}{l}\text { Principio 5: Las pruebas válidas } \\
\text { tienen una materia bien definida } \\
\text { que extrae habilidades mentales } \\
\text { humanas relevantes }\end{array}$ & $\begin{array}{l}\text { Las personas exhiben sus habilidades de razonamiento } \\
\text { mientras resuelven problemas dentro de un área temática } \\
\text { determinada. Como tal, la validez de una prueba depende tanto } \\
\text { del contenido que muestrea como de las habilidades mentales } \\
\text { humanas que provoca. }\end{array}$ \\
\hline $\begin{array}{l}\text { Principio 6: IE es una inteligencia } \\
\text { amplia }\end{array}$ & $\begin{array}{l}\text { Las inteligencias amplias se dividen en subclases como el } \\
\text { reflejo de las capacidades funcionales básicas del cerebro, } \\
\text { como la velocidad de procesamiento mental y el alcance de } \\
\text { la memoria de trabajo, la inteligencia auditiva y la inteligencia } \\
\text { táctil }\end{array}$ \\
\hline $\begin{array}{l}\text { Principio 7: IE es un miembro de } \\
\text { la clase de inteligencias amplias } \\
\text { centradas en el procesamiento de } \\
\text { información rápida }\end{array}$ & $\begin{array}{l}\text { El ser humano piensa regularmente rápido ante una acción } \\
\text { que se le presenta, pero no siempre actúa con la misma } \\
\text { velocidad. Cuando si responde rápido y no hizo un análisis } \\
\text { de la información suele equivocarse en la toma de decisiones } \\
\text { aplicada. Por ello, pensar claramente sobre los sentimientos, } \\
\text { la personalidad y los grupos sociales, las personas pueden } \\
\text { evaluar, hacer frente y predecir mejor las consecuencias de } \\
\text { sus propias acciones y el comportamiento de las personas } \\
\text { que los rodean. }\end{array}$ \\
\hline
\end{tabular}

Fuente: Elaboración propia con base en Mayer et al (2016)

De manera que, en la tabla 3 muestra de manera precisa puntos a evaluar sobre la medición de la IE de un individuo, o para esta investigación, de un emprendedor y analizar si con la suma de todos estos principios logra hacer un impacto directo en la PE y con ello, posteriormente, en la consolidación de la empresa.

En este tenor y con base en lo expuesto por Puertas-Molero et al (2020), la IE representa un elemento clave en el bienestar social y mental de quien la desarrolla, lo cual genera un impacto positivo en la toma de decisiones, siendo esto una parte esencial para los emprendedores.

En este sentido, se plantea la segunda hipótesis de estudio en esta investigación:

H2. La inteligencia emocional del emprendedor influye positivamente en la importancia percibida sobre la planeación estratégica

Para mayor comprensión de las hipótesis planteadas, se muestra a continuación la figura 2. 
Figura 2. Modelo estadístico del estudio

Conocimientos y capacidades

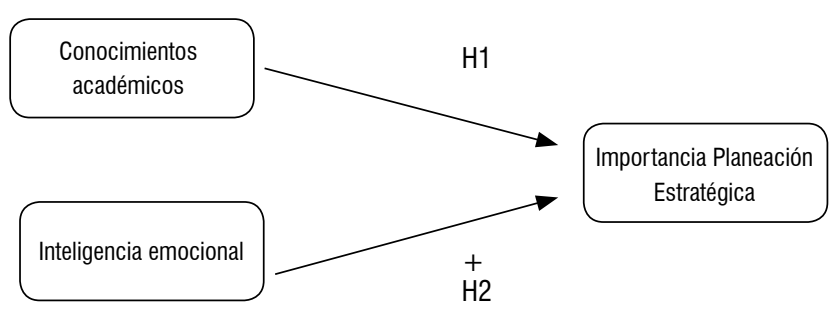

Fuente: elaboración propia

\section{METODOLOGÍA}

Debido a la naturaleza de este trabajo, la información ha sido recabada, en un primer momento, a través de una revisión de literatura misma que de acuerdo con Bernal (2010) consiste en un análisis de la información escrita de un tema específico, con el propósito de establecer relaciones, diferencias o posicionamientos del estado actual del conocimiento del sujeto que se encuentra en estudio.

Con base en el análisis de la literatura, se diseñó un instrumento de medición de las variables de estudio y fue aplicado a 133 emprendedores restauranteros de la ZMG a través de encuestas impresas y de la herramienta Google Forms., para poder brindar resultados contundentes de lo investigado para este trabajo se realizó un proceso estadístico inferencial utilizando el software SPSS.

Una vez obtenidos los datos, se procedió a realizar un Análisis Factorial Exploratorio (AFE) con el fin de obtener los factores a analizar en los modelos de estadística inferencial. Se comprobó la adecuación muestral mediante el análisis del coeficiente Kaiser, Meyer y Olkin (KMO) y se revisó la varianza explicada de cada una de las variables independientes (CA e IE) así como la agrupación de ítems en la matriz de componentes rotados.

Posteriormente, se realizó el mismo procedimiento para obtener la factorización de la variable dependiente (importancia percibida de la $\mathrm{PE}$ ) revisando el ajuste de los mismos indicadores KM0>.700 y la varianza explicada del factor.

Al contar con las escalas definidas de cada una de las variables independientes y la variable dependiente, se procedió a calcular la fiabilidad de dichas escalas mediante un análisis de alfa de Cronbach, el cual de acuerdo con Cervantes (2005) aporta un nivel de confianza a un cuestionario con la condicionante que el resultado sea un indicador $\alpha>.700$.

Se realizó un modelo de correlaciones bivariadas, a fin de conocer la relación causal entre las variables y la forma en que el comportamiento de una impacta sobre el comportamiento de la otra. 
Finalmente se calculó un modelo de regresiones lineales con la finalidad de determinar la capacidad predictiva de las variables independientes (CA e IE) sobre la variable dependiente (importancia percibida sobre la PE). En la tabla 4 se muestra el instrumento utilizado en este trabajo, el que considera una escala de 1 a 7.

Tabla 4. Instrumento de recolección de datos

\begin{tabular}{|l|}
\hline \multicolumn{1}{|c|}{ Planeación estratégica } \\
\hline Para mi fue importante establecer una planeación a detalle cuando inicié mi empresa \\
\hline Considero que planear detalladamente da por hecho que elevará los ingresos mi empresa \\
\hline $\begin{array}{l}\text { Tomo en cuenta los factores externos (políticos y económicos) de la empresa como } \\
\text { relevantes }\end{array}$ \\
\hline $\begin{array}{l}\text { En mi empresa es necesario tener la estrategia por escrito para que todo el equipo sepa } \\
\text { la meta a la que se dirige la misma }\end{array}$ \\
\hline Considero importante revisar la planeación hecha periódicamente para posibles ajustes \\
\hline Desde mi perspectiva los casos pasados de mi empresa aportan a mi planeación \\
\hline Los casos pasados y actuales de otras empresas aportan a mi planeación \\
\hline Frecuentemente estoy al pendiente de lo que sucede en el mercado de mi industria \\
\hline Analizo regularmente las tendencias de mis clientes \\
\hline Enfoco mayormente las metas a largo plazo que las del corto plazo \\
\hline
\end{tabular}

\begin{tabular}{|l|}
\hline \multicolumn{1}{|c|}{ Conocimientos académicos } \\
\hline Los cursos académicos tomados me han dado una visión sobre el emprendimiento \\
\hline Los cursos académicos tomados me han hecho querer ser auto empleado \\
\hline $\begin{array}{l}\text { Los cursos académicos tomados me han hecho sentir que el término emprendedor } \\
\text { encaja en mí }\end{array}$ \\
\hline Gracias a los cursos académicos tomados me encantaría verme como emprendedor \\
\hline Los cursos académicos tomados me hacen sentir listo para emprender \\
\hline Debido a los cursos académicos tomados sé de los riesgos de emprender \\
\hline $\begin{array}{l}\text { Después de haber tomado cursos académicos, he comenzado a conocer a } \\
\text { emprendedores de éxito y a admirar a los mismos }\end{array}$ \\
\hline $\begin{array}{l}\text { Los cursos académicos tomados me han brindado herramientas para simular la } \\
\text { creación, desarrollo y consolidación de la empresa }\end{array}$ \\
\hline $\begin{array}{l}\text { Después de haber tomado cursos académicos sé que hay más ventajas de ser } \\
\text { emprendedor que de no serlo }\end{array}$ \\
\hline $\begin{array}{l}\text { Los cursos académicos tomados me han incentivado a leer con mayor frecuencia } \\
\text { noticias y libros sobre emprendimiento }\end{array}$ \\
\hline \multicolumn{1}{|c|}{ Inteligencia emocional } \\
\hline La mayoría del tiempo, tengo la capacidad de identificar y separar mis emociones \\
\hline Estoy consciente del impacto de mi humor en otras personas \\
\hline Sé el impacto que generan las emociones en mis propias acciones \\
\hline Reconozco mis propias fortalezas y debilidades \\
\hline Suelo tener control de mi mismo(a) cuando estoy enojado(a) \\
\hline $\begin{array}{l}\text { Suelo mantener el mismo parecer sobre una idea a pesar de cambios pequeños en el } \\
\text { entorno }\end{array}$ \\
\hline Suelo auto motivarme para dar siempre lo mejor de mi \\
\hline Se me facilita lidiar con el estrés \\
\hline Me describo a mi mismo como un muy buen negociador(a) \\
\hline Difícilmente me envuelvo en cosas que después se me dificulta desprenderme \\
\hline
\end{tabular}

Fuente: elaboración propia 


\section{Mediciones y resultados}

Como se mencionó anteriormente se comenzó el análisis estadístico con el Análisis Factorial Exploratorio y podemos observar en la tabla 5, que el indicador, KM0 es de .907, con un nivel de significancia de $<.001$ en la prueba de esfericidad de Bartlett. Lo que indica claramente que la muestra es adecuada y se puede continuar con el proceso estadístico, ya que según Landero y González (2014) el mínimo requerido en el KM0 es de .700.

Tabla 5.- Análisis de adecuación muestral KMO variables independientes

\begin{tabular}{|l|l|l|}
\hline \multicolumn{2}{|l|}{ Medida Kaiser-Meyer-Olkin de adecuación de muestreo } & .907 \\
\hline \multirow{3}{*}{ Prueba de esfericidad de Barlett } & Aprox. Chi-cuadrado & 1156.410 \\
\cline { 2 - 3 } & gl & 91 \\
\cline { 2 - 3 } & Sig. & .000 \\
\hline
\end{tabular}

Fuente: Elaboración propia a través de SPSS

El siguiente paso en el proceso de análisis es la factorización de las variables mediante una reducción de dimensiones y se obtuvieron los siguientes resultados:

En la tabla 6, se puede observar la matriz de componentes rotados, misma que brinda información pertinente en el sentido que agrupa y califica si los ítems dados pertenecen al mismo componente con el objetivo de examinar que la pregunta 0 ítem no tenga igual significado al mismo tiempo que responde a otro ítem. Se obtiene una agrupación adecuada y congruente con la teoría y el diseño del cuestionario.

Tabla 6.- Análisis de matriz de componentes rotados

\begin{tabular}{|c|c|c|}
\hline \multirow{2}{*}{} & \multicolumn{2}{|c|}{ Componente } \\
\hline & $\mathbf{1}$ & $\mathbf{2}$ \\
\hline CA1 & .835 & \\
\hline CA2 & .721 & \\
\hline CA3 & .884 & \\
\hline CA & .888 & \\
\hline CA5 & .858 & \\
\hline CA6 & .842 & \\
\hline CA7 & .823 & \\
\hline CA8 & .827 & \\
\hline CA9 & .853 & \\
\hline CA10 & .758 & \\
\hline IE1 & & .741 \\
\hline IE5 & & .775 \\
\hline IE6 & & .660 \\
\hline IE8 & & .653 \\
\hline
\end{tabular}

Fuente: elaboración propia a través de SPSS 
Así como fue mostrado en el apartado anterior los resultados de KMO y matriz de componentes rotados, a continuación se realiza lo mismo en los respectivos a la variable dependiente de esta investigación; en este caso, solo se mostrará una matriz de componentes (no rotados) pues al ser una sola variable a analizar, este estudio no puede llevarse a cabo.

Como puede ser observado en la tabla 7 el indicador de KMO es la PE (variable dependiente) es de .812 lo cual cumple con los parámetros mencionados con anterioridad,

Tabla 7.- Análisis de adecuación muestral KMO variable dependiente

\begin{tabular}{|l|l|l|}
\hline Medida Kaiser-Meyer-0lkin de adecuación de muestreo & .812 \\
\hline \multirow{2}{*}{$\begin{array}{l}\text { Prueba de esfericidad de } \\
\text { Barlett }\end{array}$} & Aprox. Chi-cuadrado & 398.604 \\
\cline { 2 - 3 } & gl & 15 \\
\cline { 2 - 3 } & Sig. & .000 \\
\hline
\end{tabular}

Fuente: elaboración propia a través de SPSS

Continuando con lo respectivo a esta variable, se pueden observar un componente único de la PE en la tabla 8, complementando el nivel de solidez que cumple esta variable.

Tabla 8. - Análisis de matriz de componentes

\begin{tabular}{|c|c|}
\hline \multirow{2}{*}{} & Componente \\
\cline { 2 - 2 } & 1 \\
\hline PE4 & .570 \\
\hline PE5 & .769 \\
\hline PE6 & .862 \\
\hline PE7 & .824 \\
\hline PE8 & .802 \\
\hline PE9 & .772 \\
\hline
\end{tabular}

Fuente: elaboración propia a través de SPSS

Ahora, para verificar el nivel de fiabilidad de estos resultados presentados, se muestra la tabla 9 donde se presentan los valores brindados por el alfa de Cronbach de estos tres indicadores. Como se puede observar, el porcentaje del alfa de Cronbach en los CA y la PE es alto y por ende fiable; en lo que respecta la PE al ser un indicador de corte más cualitativo, Taber (2017) afirma que el nivel del alfa de Cronbach suele disminuir del nivel ideal que suele ser de un mínimo de .700 por lo que es considerado totalmente fiable el uso del indicador de IE para este trabajo de investigación. 
Tabla 9.- Alfa de Cronbach indicadores

\begin{tabular}{|l|l|}
\hline \multicolumn{1}{|c|}{ Variable } & \multicolumn{1}{c|}{ Alfa de Cronbach } \\
\hline Conocimientos académicos & .956 \\
\hline Planeación estratégica & .850 \\
\hline Inteligencia emocional & .677 \\
\hline
\end{tabular}

Fuente: elaboración propia de acuerdo con los datos de SPSS

Con la información brindada anteriormente, se muestra a continuación en la tabla 10 las correlaciones que tienen las variables independientes de CA por parte del emprendedor, así como la IE con la variable dependiente la cual es la PE.

Tabla 10.- Correlaciones entre variables

\begin{tabular}{|l|l|c|c|c|}
\hline & & PlanEstra & ConocAcadem1 & IntelEmoc1 \\
\hline PlanEstra & Correlación de Pearson & 1 & $.444^{\star *}$ & $.481^{\star *}$ \\
\hline & Sig. (bilateral) & & .000 & .000 \\
\hline & $\mathrm{N}$ & 133 & 133 & 133 \\
\hline ConAcadem11 & Correlación de Pearson & $.444^{\star \star}$ & 1 & $.291^{\star *}$ \\
\hline & Sig. (bilateral) & .000 & & .001 \\
\hline & $\mathrm{N}$ & 133 & 133 & 133 \\
\hline IntelEmoc1 & Correlación de Pearson & $.481--$ & $.291^{\star *}$ & 1 \\
\hline & Sig. (bilateral) & .000 & .001 & \\
\hline & $\mathrm{N}$ & 133 & 133 & 133 \\
\hline
\end{tabular}

Fuente: elaboración propia a través de SPSS

Al observar el nivel de correlación existente entre variables, se muestra que los CA influyen en un $44.4 \%$ mientras que la IE en un $48.1 \%$ en una PE efectiva en una organización. La información recabada aporta a esta investigación el hallazgo que actitudes como el control del estrés, o de las emociones en situaciones difíciles puede dar mejores resultados en la PE.

En las siguientes tablas, se observa los resultados de las regresiones implementadas obteniendo de resultados relevantes para este estudio el $\mathrm{R}^{2}$ ajustado así como las cargas de beta y la significancia de las variables independientes.

En la tabla 11 se muestra con el primer resultado de las regresiones un dato de $\mathrm{R}^{2}$ ajustado el cual menciona que el modelo explica en un $32.8 \%$ el fenómeno a analizar, lo cual es una estadística importante pues es menester comprender que tanto Ios CA como la IE son solo dos de diversos factores que pueden aportar información de cómo una PE eficiente y eficaz pueda implementarse. 
Tabla 11.- Resumen del modelo para $\mathrm{R}^{2}$ ajustado

\begin{tabular}{|c|c|c|c|c|}
\hline Modelo & $\mathbf{R}$ & R cuadrado & R cuadrado ajustado & Error estándar de la estimación \\
\hline 1 & .582 & .338 & .328 & .81968690 \\
\hline
\end{tabular}

Fuente: elaboración propia a través de SPSS

Dando continuidad al análisis, en la tabla 12 se observa un nivel de significancia de ambas variables independientes de .000 lo que indica un nivel alto así como cargas estadísticas de .382 y .438 datos que no son leídos en porcentaje si no en el peso que estas variables tienen sobre la PE.

Tabla 12.- Resumen del modelo coeficiente beta y significancia

\begin{tabular}{|l|l|l|l|l|l|}
\hline & \multicolumn{2}{|l|}{$\begin{array}{l}\text { Coeficientes no } \\
\text { estandarizados }\end{array}$} & $\begin{array}{l}\text { Coeficientes } \\
\text { estandarizados }\end{array}$ & & \\
\hline Modelo & B & Desv. Error & Beta & T & Sig. \\
\hline 1 (Constante) & $9.217 \mathrm{E}-18$ & .071 & & .000 & 1.000 \\
\hline ConocAcad & .382 & .071 & .382 & 5.356 & .000 \\
\hline IntelEmoc & .438 & .071 & .438 & 6.146 & .000 \\
\hline
\end{tabular}

Fuente: elaboración propia a través de SPSS

Una vez dado estos puntos de análisis, se resalta que hay diversos factores para decir que la formación emprendedora influye en mayor proporción en la consolidación empresarial como la IE y en menor porcentaje los CA, de acuerdo con las respuestas aportadas por parte de los emprendedores no es un nivel considerable para tomar en cuenta. No obstante, esto no puede dejarse de lado, debido a como se estableció con anterioridad, la situación vivida por la pandemia de COVID-19 ha puesto la IE como una parte central que cada emprendedor debería tomar en consideración. Los niveles de MiPyMEs que han podido sobrevivir y adaptarse al entorno con los cambios demandantes que ha provocado esta pandemia han sido un factor de IE que quienes lo han aplicado han podido salir más favorables.

Siguiendo con la idea anterior, la PE representa un pilar para tomarse en consideración en mayor proporción, lo cual no significa que un emprendedor no pueda hacer ajustes a la planeación presentada pues de ello también van las características que se debe tener ante una adaptación al entorno interno y sobre todo externo de la empresa, pero sí es importante que se utilicen modelos de planeación como el marco lógico mismo que Sánchez (2007) explica que brinda un proceso con un nivel de facilidad de mayor comprensión comparado con otros modelos acentuando la planeación dicha anteriormente, así como el seguimiento y evaluación de proyectos poniendo como eje central de la investigación las actividades a realizar, los componentes, el propósito y el fin de las estrategias a implementar.

Por otro lado, se encuentra el modelo de canvas el cual de acuerdo con 0sterwalder y Pigneur (2010) está dividido en 9 secciones haciendo énfasis en la consideración 
que se debe imprimir en estos apartados para una mayor probabilidad de éxito en la empresa. Este modelo de canvas está compuesto por la propuesta de valor; segmento de clientes; canales de distribución; relación con los clientes; fuente de ingresos; recursos clave; actividades clave; socios clave y finalmente la estructura de los costos.

\section{DISCUSIÓN Y CONCLUSIONES}

Como ya se ha comentado, el conocimiento de los emprendedores en cualquier sector es un activo fundamental en el futuro de las empresas. La formación que los sujetos han obtenido y la aplicación de ese conocimiento en el desarrollo de planes estratégicos estructurados, dictarán sin duda el destino de sus proyectos empresariales en términos de supervivencia, crecimiento y en su caso consolidación.

Sin embargo, se reconoce que no todos los emprendedores consideran la PE como un elemento que influya en los planes de crecimiento y consolidación de sus proyectos, a pesar de estar demostrado una relación positiva de los mismos. Este trabajo busca reconocer si elementos cognitivos del sujeto como los CA durante su proceso educativo o los elementos de su IE, afectan de manera positiva la percepción sobre la importancia de la PE, considerándola una herramienta fundamental para la supervivencia, crecimiento y consolidación de sus proyectos empresariales.

Después de los procesos de revisión de literatura, construcción de variables y diseño e implementación del cuestionario para recoger datos, y habiendo realizado los procesos estadísticos correspondientes, se encuentra que, entre los empresarios del sector restaurantero en la ZMG, tanto los conocimientos adquiridos mediante la formación educativa, como los elementos de la IE del sujeto, tienen una capacidad explicativa en sentido positivo de la percepción de importancia que el emprendedor le da al proceso de PE, en plena consciencia de la importancia que esta tiene para la consolidación de su proyecto (véase figura 3).

Figura 3. Modelo estadístico de resultados

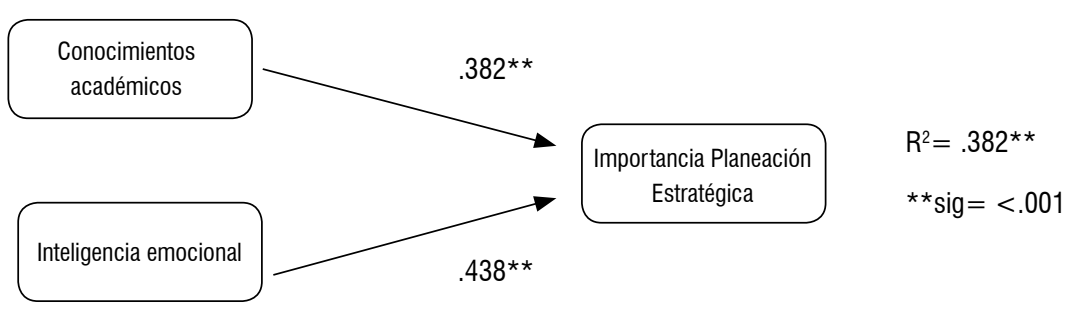

Fuente: Elaboración propia 
A manera de conclusión se establece que una vez demostrada mediante la revisión de literatura, la importancia de la PE en la supervivencia, crecimiento y consolidación de las empresas, y una vez probada mediante métodos estadísticos la influencia positiva de factores cognitivos como la IE y los CA en el reconocimiento que el emprendedor da a la importancia de la $\mathrm{PE}$, se plantea que, cuando se habla de estrategias para buscar la consolidación de proyectos de emprendimiento, la formación académica que el sujeto reciba será esencial.

Un resultado interesante es, a pesar de que el proceso de PE está relacionado con una estrategia de capacitación y formación técnica para ser realizado, Ios estadísticos del modelo muestran un mayor peso explicativo por parte de la IE que de los mismos CA en la importancia dada a la PE por parte del emprendedor.

Esto puede explicarse también, puesto que al igual que la IE influye en la toma de decisiones, muchos emprendedores asocian directamente la PE con ese mismo proceso de toma de decisiones, sin embargo, este resultado constituye un interesante tema para investigaciones futuras.

En este orden de ideas se comprueban ambas hipótesis de investigación. Por una parte, los CA afectan de manera positiva la percepción de importancia de la PE en los emprendedores del sector restaurantero de la ZMG. (Hipótesis 1).

Por otra parte, se demuestra que la IE afecta también de manera positiva la percepción de importancia de la PE en los mismos emprendedores del sector restaurantero de la ZMG. (Hipótesis 2).

Se reconoce de igual forma la importancia de la IE en una época donde la PE requiere ser revisada y ajustada a las nuevas realidades que las empresas enfrentan por motivo de la crisis sanitaria y económica adherente a la pandemia COVID-19.

Si bien es cierto que la nueva normalidad para las empresas trae consigo una reorientación de los objetivos empresariales originales, también es verdad que la IE en la toma de decisiones tendrá un papel importante que jugar.

La IE permitirá a los emprendedores y empresarios, redirigir el rumbo de sus proyectos y actualizar su PE para ajustarse a la nueva ruta de acción en esta normalidad post COVID-19.

Por ello, esta investigación brinda información puntual sobre la estructuración de una PE a través de factores como los CA y la IE para que con ello el nivel de consolidación de las MiPyMEs pueda lograrse y conservarse en el mercado. Sin duda alguna, esto beneficia e impacta directamente en de una sociedad a través de empleos y derrama económica, puesto que un área geográfica tan importante en México como lo es la ZMG le es sumamente necesario tener y presentar dichos resultados como lugar próspero y así motivar a mayor inversión nacional y extranjera. 


\section{LIMITACIONES Y FUTURAS LÍNEAS DE INVESTIGACIÓN}

Como limitaciones del estudio se menciona el presente trabajo constituye una parte preliminar de una investigación más compleja referente a una mayor cantidad de capacidades cognitivas y su relación al proceso integral de consolidación empresarial. Por tanto, se plantean los presentes resultados como un hallazgo inicial de un modelo mayor, con lo cual se reconocen algunas brechas tanto en la literatura como en la interpretación del fenómeno.

Asimismo, se menciona que el número de encuestas realizadas fue a 133 restauranteros lo cual implica un porcentaje bajo respecto al total del universo puesto como ha sido mencionado, este es un proyecto parte de una investigación con un nivel profundidad mayor.

También se resalta como futura línea de investigación, el indagar más sobre los motivos que hacen que la IE tenga mayor peso incluso que los CA sobre la importancia percibida de la $\mathrm{PE}$, un elemento que tradicionalmente se ha asociado precisamente con la educación y la capacitación de los emprendedores. De la misma manera, resaltar que esta investigación del sector restaurantero pueda ser replicable en otras industrias con el objetivo que aporte información pertinente a las mismas, ya que valores del emprendedor como sus CA e IE y su impacto en la PE como factor de consolidación pueden aplicar en cualquier otro sector económico. 


\section{REFERENCIAS}

BARTKOWIAK, G., KRUGIEŁKA, A., DACHOWSKI, R., GAŁEK, K., \& KOSTRZEWA-DEMCZUK, P. (2020). Attitudes of Polish Entrepreneurs towards $65+$ Knowledge Workers in the Context of Their Pro-Social Attitude and Organizational Citizenship Behavior. Sustainability, 12(13), 1-24.

BERNAL, C. (2010). Metodología para la investigación. Bogotá: Pearson.

BOLIN, S. (2015). Behaviors contributing to native American business success. Minneapolis: Walden University.

BOLISANI, E., \& BRATIANU, C. (2018). Emergent knowledge strategies: strategic thinking in knowledge management. Cham: Springer.

CAMARA NACIONAL DE LA INDUSTRIA RESTAURANTERA Y DE ALIMENDOS CONDIMENTADOS DE JALISCO. (22 de Octubre de 2020). CANIRAC. Obtenido de canirac-jalisco.com: https://canirac-jalisco.com/2020/02/13/ jalisco-tiene-34204-restaurantes-y-cafeterias-y-1921-florerias-parafestejar-el-dia-del-amor-y-la-amistad/

CARAYANNIS, E. (2011). Nature Vs. Nurture: Who is Interested in Entrepreneurship Education? A Study of Business and Technology Undergraduates Based on Social Cognitive Career Theory. Washington D.C.: The Faculty of the School of Business of The George Washington University.

CARTER, S. (2015). Emotional intelligence: a qualitative study of the development of emotional intelligence of community college students enrolled in a ledership development program. Fort Collins : Colorado State University.

CERVANTES, V. (2005). Interpretaciones del coheficiente alpha de Cronbach. Avances en medición, 9-28.

CHURCHILL, N., \& LEWIS, V. (1983). The Five Stages of Small Business Growth. Harvard Business Review, 1-11.

COLEMAN, M. (1994). An exploration of students' and business persons' ratings of the importance of writing skills for business success. Michigan: Western Michigan University.

CORREDOIRA, R., \& KIMBERLY, J. (2006). Industry evolution through consolidation: implications for addiction treatment. Journal of Subtance Abuse Treatment, 31(3), 255-265.

FORBES. (16 de Septiembre de 2020). OCDE prevé mayor caída del PIB de México para 2020. Recuperado el 19 de Octubre de 2020, de Forbes. 
com.mx: $\quad$ https://www.forbes.com.mx/economia-ocde-prevision-pibmexico-2020/

FRETSCHNER, M., \& WEBER, S. (2013). Measuring and understanding the effects of entrepreneurial awareness education. Journal of Small Business Management, 51(3), 410-428.

FURNHAM, A. (2012). Emotional intelligence. New Perspectives and Applications, 1-28.

GAMBARDELlA, A., \& FLORENCE, M. (2015). Using What You Know: Patented Knowledge in Incumbent Firms and Employee Entrepreneurship. Organization Science, 26(2), 456-474.

GLOBAL ENTREPRENEURSHIP MONITOR. (2019). Comportamiento $y$ actitudes empresariales. Obtenido de gemconsortium.org: https://www. gemconsortium.org/economy-profiles/mexico-2

GOLEMAN, D., BOYATZIS, R., \& MCKEE, A. (2013). Primal leadership: unleashing the power of emotional intelligence. Boston: Harvard Business Review.

GREEN, T., GOLD, S., \& PRICE, T. (2014). Entrepreneurial intentions: making the case for entrepreneurship education. Minneapolis: Capella University.

GUADARRAMA, E., \& ROSALES, E. (2015). Marketing relacional: valor, satisfacción, lealtad y retención del cliente. Análisis y reflexión teórica. Ciencia y Sociedad, 40(2), 307-340.

HAYTER, C., \& LINK, A. (2018). Why do knowledge-intensive entrepreneurial firms publish their innovate ideas? Academy of Management Perspectives, 32(1), 141-155.

INSTITUTO NACIONAL DE ESTADÍSTICA Y GEOGRAFÍA. (23 de Julio de 2020). EI INEGI presenta resultados del impacto del COVID-19 en la actividad económica y el mercado laboral. Recuperado el 12 de 0ctubre de 2020, de inegi.org.mx: https://www.inegi.org.mx/contenidos/saladeprensa/ boletines/2020/0trTemEcon/COVID-ActEco.pdf

INSTITUTO NACIONAL DE ESTADÍSTICA Y GEOGRAFÍA. (25 de marzo de 2021). Directorio estadístico nacional de unidades económicas. Obtenido de inegi.org.mx: https://www.inegi.org.mx/app/mapa/denue/default.aspx

INSTITUTO NACIONAL DE ESTADÍSTICA Y GEOGRAFÍA. (8 de abril de 2020). Esperanza de vida de los negocios. Recuperado el 8 de abril de 2020, de inegi.org.mx: https://www.inegi.org.mx/temas/evnm/default. html\#Informacion_general 
JONCKHEERE, M. (2015). Independent Management Consulting Business Success. Malibú: Pepperdine University .

KAISER, E. (2011). Self-efficacy, burnout, and success of small business owners in New York. Minneapolis: Capella University.

KAYUNI, H. (2016). Strategic planning in the Malawi public sector: potencial tool for progress or regression? Public Organization Review, 373-391.

LANDEROS, R., \& GONZÁLEZ, M. (2014). Estadística con SPSS y metodología de la investigación. Monterrey: Universidad Autónoma de Nuevo Léon.

LUSSIER, R. (1992). Contributing factors to success versus failure of the startup business: startup business success versus failure prediction models. West Haven: University of New Haven.

MATA-LOERA, M. E. (18 de Mayo de 2020). Cada vez más crítica la situación de restauranteros por cuarentena. Obtenido de gaceta.udg.mx: http:// www.gaceta.udg.mx/cada-vez-mas-critica-situacion-de-restauranterospor-cuarentena/

MAYER, J., CARUSO, D., \& SALOVEY, P. (2016). The Ability Model of Emotional Intelligence: Principles and Updates. Emotion Review, 1-11.

MICROSOFT LATINOAMÉRICA. (12 de Agosto de 2020). La tecnología como pieza clave para las PyMEs después de la pandemia. Recuperado el 13 de Octubre de 2020, de news.microsoft.com: https://news.microsoft. com/es-xl/la-tecnologia-como-pieza-clave-para-las-pymes-despues-dela-pandemia/

ORDAZ-DÍAZ, A. (19 de Diciembre de 2019). Pide Coparmex más apoyo para Pymes y proyectos de innovación juvenil en CDMX. Recuperado el 19 de Octubre de 2020, de Forbes.com.mx: https://www.forbes.com.mx/pidecoparmex-mas-apoyo-a-pymes-y-a-proyectos-de-innovacion-juvenil-encdmx/

OSTERWALDER, A., \& PIGNEUR, Y. (2010). Business model generation: a handbook for visionaries, game changers, and challengers. Hoboken: Wiley.

PUERTAS-MOLERO, P., ZURITA-ORTEGA, F., CHACÓN-CUBEROS, R., CASTROSÁNCHEZ, M., RAMÍREZ-GRANIZO, I., \& GONZÁLEZ-VALERO, G. (2020). La inteligencia emocional en el ámbito educativo: un meta-análisis. anales de la psicología, 36(1), 84-91.

RONMARK , T. (2005). Evaluating the role collaborative planning in BC's parks and protected areas management planning proces. Surrey: Simon Fraser University. 
SÁNCHEZ, N. (2007). El marco lógico. Metodología para la planificación, seguimiento y evaluación de proyectos. Visión Gerencial, 328-343.

SCHOFIELD, R. (2015). Relationship Between Bootstrap Financing, Number of Employees, and Small Business Success. Minneapolis: Walden University.

SENIOR, A. (2016). Achieving longevity in business: determinants of small business success. Florida: Lynn University.

TABER, K. (2017). The Use of Cronbach's Alpha When Developing and Reporting Research Instruments in Science Education. Research in Science Education, 1-24.

THE EUROPEAN COMMISSION. (10 de septiembre de 2006). Entrepreneurship Education in Europe: Fostering Entrepreneurial Mindsets through Education and Learning. Recuperado el 8 de noviembre de 2019, de ec.europa.eu: https://ec.europa.eu/growth/content/entrepreneurship-education-europefostering-entrepreneurial-mindsets-through-education-and_en

WALTER, J., \& PANDO, D. (2014). Planificación estratégica nuevos desafíos y enfoques en el ámbito público. Buenos Aires: SAAP.

WALTERS, S. (2018). Emotional intelligence and leader development: measuring trait emotional intelligence scores of mid-career commissioned U.S. army officers. Bowling Green: Western Kentucky University.

WEN-HSIN, A., JUNG, B., \& POURJALADI, H. (2015). Does international accounting standard no. 27 improve investment efficiency? Journal of Accounting, Auditing \& Finance, 30(4), 484-508. 